\title{
Association Analysis between Genes' Variants for Regulating Mitochondrial Dynamics and Fasting Blood Glucose Level
}

\author{
Dongju Jung ${ }^{1}$ and Hyun-Seok Jin ${ }^{1, \dagger}$ \\ Department of Biomedical Laboratory Science, College of Life and Health Sciences, \\ Hoseo University, Asan, Chungnam 31499, Korea
}

\begin{abstract}
Maintenance of fasting blood glucose levels is important for glucose homeostasis. Disruption of feedback mechanisms are a major reason for elevations of glucose level in blood, which is a risk factor for type 2 diabetes mellitus that is mainly caused by malfunction of pancreatic beta-cell and insulin. The fasting blood glucose level has been known to be influenced by genetic and environmental factors. Mitochondria have many functions for cell survival and death: glucose metabolism, fatty acid oxidation, ATP generation, reactive oxygen species (ROS) metabolism, calcium handling, and apoptosis regulation. In addition to these functions, mitochondria change their morphology dynamically in response to multiple signals resulting in fusion and fission. In this study, we aimed to examine association between fasting blood glucose levels and variants of the genes that are reported to have functions in mitochondrial dynamics, fusion and fission, using a cohort study. A total 416 SNPs from 36 mitochondrial dynamics genes were selected to analyze the quantitative association with fasting glucose level. Among the 416 SNPs, 4 SNPs of PRKACB, 13 SNPs of PPP3CA, 6 SNPs of $P A R K 2$, and 3 SNPs of GDAP1 were significantly associated. In this study, we were able to confirm an association of mitochondrial dynamics genes with glucose levels. To our knowledge our study is the first to identify specific SNPs related to fasting blood glucose level.
\end{abstract}

Key Words: Mitochondrial dynamics, Genetic variation, SNP, Glucose level, Association study

\section{INTRODUCTION}

Maintenance of fasting blood glucose levels is important for glucose homeostasis. Disruption of feedback mechanism is a major reason for elevations of glucose level in blood, which is a risk factor for type 2 diabetes mellitus that is mainly caused by malfunction of pancreatic beta-cell and insulin (Prokopenko et al., 2009; Mason et al, 2007; Jin et al, 2014). Fasting blood glucose levels are influenced by genetic and environmental factors, but genetic factors that contribute to heritability remain elusive (Watanabe et al., 1999).

Mitochondria are essential organelles in most eukaryotic cells for maintaining cellular homeostasis. Performances of mitochondria contribute to cell survival and death: glucose metabolism, fatty acid oxidation, ATP generation, reactive oxygen species (ROS) metabolism, calcium handling, and apoptosis regulation (McBride et al., 2006; In et al., 2013). Mitochondrion is also an organelle that is continually remodeled by fusion and fission in response to a multitude of signals (Chan, 2006; Hoppins et al., 2007). Therefore, morph-

\footnotetext{
*Received: September 4, 2016 / Revised: September 29, 2016 / Accepted: September 30, 2016

${ }^{\dagger}$ Corresponding author: Hyun-Seok Jin. Department of Biomedical Laboratory Science, College of Life and Health Sciences, Hoseo University, Asan, Chungnam 31499, Korea.

Tel: +82-41-540-9968, Fax:+82-41-540-9997, e-mail: jinhs@hoseo.edu

(C) The Korean Society for Biomedical Laboratory Sciences. All rights reserved.

(c) This is an Open Access article distributed under the terms of the Creative Commons Attribution Non-Commercial License (http://creativecommons.org/licenses/by-nc/3.0/) which permits unrestricted non-commercial use, distribution, and reproduction in any medium, provided the original work is properly cited.
} 
ology of mitochondria is cycling between fusion and fission. This phenomenon is referred to as "mitochondrial dynamics", and it has been known that many regulating proteins are involved in mitochondrial dynamics (Chan, 2006; Hoppins et al., 2007). Currently, the regulating proteins are divided to five groups: 1) fusion shaping, 2) fission shaping, 3) fusion regulation, 4) fission regulation, and 5) fusion and fission regulation (Chan, 2006; Hoppins et al., 2007; Cerveny et al., 2007; Benard \& Karbowski, 2009; Otera et al., 2013). In this study, we examined the association between fasting blood glucose levels and variants of the genes that are involved in mitochondrial dynamics, fusion and fission, using a cohort study.

\section{MATERIALS AND METHODS}

\section{Subjects}

Korean subjects within the Korean Association REsource (KARE) study were described in more detail by another study (Cho et al., 2009). Briefly, the participants were recruited from two community-based epidemiological cohorts; the rural community of Ansung city and the urban community of Ansan city. The cohorts are composed of 8842 people (4,183 men and 4,659 women) aged from 40 to 69 years old. Both cohorts were used for studies conducted in 2001 as part of the Korean Genome Epidemiology Study (KoGES). For accurate analysis of blood glucose, 1291 subjects who had been treated with drugs were excluded and the remaining 7551 subjects (3,747 men and 3,804 women) were finally investigated. The basic characteristics of the subjects are described in Table 2. Fasting glucose levels of the subject were obtained from the data in the KARE study. This study has been approved by the Institutional Review Board of the Korean National Institute of Health (KNIH). Written informed consent was obtained from all of subjects.

\section{Genotyping and selection of SNPs}

The detailed genotyping, quality control processes and quantitative traits were described in a previous report (Cho et al., 2009). Briefly, DNA samples were isolated from all of the participants' peripheral blood and genotyped using the Affymetrix Genome-Wide Human SNP array 5.0 (Affy- metrix, Inc., Santa Clara, CA, USA). The accuracy of the genotyping was calculated by Bayesian Robust Linear Modeling using the Mahalanobis Distance (BRLMM) algorithm (Rabbee \& Speed, 2006). Samples that had genotyping accuracies were lower than $98 \%$, high missing genotype call rates $(\geq 4 \%)$, high heterozygosity $(>30 \%)$, or gender biases were excluded.

We selected candidate regulating genes for mitochondrial dynamics from several review papers (Chan, 2006; Hoppins et al., 2007; Cerveny et al., 2007; Benard and Karbowski, 2009; Otera et al., 2013). These selected 47 genes are listed in Table 1. For selection of the SNPs from the KARE data, we included gene boundary region - $5 \mathrm{~kb}$ upstream and downstream of the first and last exons, respectively - according to NCBI human genome build 36 . Through the search processes, we were able to collect the 416 SNPs out of the 36 genes (Table 1). The clinical information and genotype data that we used were graciously provided by the Center for Genome Science, KNIH, Korea Center for Disease Control (KCDC).

\section{Statistical analysis}

Most of the statistical analyses were performed using PLINK version 1.07 (http://pngu.mgh.harvard.edu/ purcell /plink) and PASW Statistics version 18.0 (SPSS Inc., Chicago, IL, USA). The 417 SNPs selected from 37 genes were analyzed for linear regression using fasting blood glucose level as a quantitative trait. All association tests were performed under the additive genetic model. Age, gender, body mass index (BMI) and cohorts were included as covariates in the analyses. Standard statistical significance $(P<0.05)$ was determined by the two-tailed Student's $t$-test between males and females.

\section{RESULTS}

\section{Selection of SNPs of the regulating genes for mitochon-} drial dynamics out of KARE data

In this association study, we collected the 47 genes that had regulating functions toward mitochondrial dynamics (fusion, fission, fusion-regulation, fission-regulation and fusion fission-regulation) based on the current literature. 
Table 1. Information about the SNPs in the mitochondrial dynamics regulating genes

\begin{tabular}{|c|c|c|c|c|c|}
\hline Function & Gene & Gene product & Gene description & Chr. & $\begin{array}{l}\text { No. of } \\
\text { SNPs* }\end{array}$ \\
\hline \multirow[t]{3}{*}{ Fusion-shaping } & $M F N 1$ & Mfn1 & Mitofusin 1 & 3 & 4 \\
\hline & MFN2 & Mfn2 & Mitofusin 2 & 1 & 1 \\
\hline & $O P A 1$ & Opa1 & Optic atrophy 1 & 3 & 13 \\
\hline \multirow[t]{3}{*}{ Fission-shaping } & $D N M 1 L$ & Drp1 & Dynamin 1-like & 12 & 12 \\
\hline & FIS1 & Fis1 & Fission 1 homolog & 7 & 0 \\
\hline & $M F F$ & Mff & Mitochondrial fission factor & 2 & 2 \\
\hline \multirow[t]{12}{*}{ Fusion-regulation } & $A F G 3 L 1$ & AFG3L1 & AFG3 ATPase family gene 3 -like $1 / \mathrm{m}$-AAA protease & 16 & 0 \\
\hline & $A F G 3 L 2$ & AFG3L2 & AFG3 ATPase family gene 3-like 2/m-AAA protease & 18 & 6 \\
\hline & $B C L 2 L 1$ & Bcl-xL & BCL2-like 1 & 20 & 0 \\
\hline & LETM1 & LETM1 & $\begin{array}{l}\text { Leucine zipper-EF-hand containing transmembrane } \\
\text { protein } 1\end{array}$ & 4 & 1 \\
\hline & $M I B 1$ & MIB & Mindbomb homolog 1 & 18 & 6 \\
\hline & OMAl & OMA1 & OMA1 homolog, zinc metallopeptidase & 1 & 12 \\
\hline & $P A R L$ & Parl & Presenilin associated, rhomboid-like & 3 & 4 \\
\hline & PHB2 & Prohibitin 2 & Prohibitin 2 & 12 & 1 \\
\hline & PLD6 & MitoPLD & Phospholipase D family, member 6 & 17 & 0 \\
\hline & $S P G 7$ & Paraplegin & Spastic paraplegia $7 / \mathrm{m}-\mathrm{AAA}$ protease & 16 & 7 \\
\hline & STOML2 & SLP-2 & Stomatic (EPB72)-like 2 & 9 & 1 \\
\hline & YME1L1 & Yme1L & YME1-like 1 & 10 & 4 \\
\hline \multirow[t]{21}{*}{ Fission-regulation } & $B C L 2 L 2$ & Bcl-w & BCL2-like 2 & 14 & 1 \\
\hline & CAMKK1 & CaM-kinase 1 & $\begin{array}{l}\text { Calcium/calmodulin-dependent protein kinase kinase } 1 \text {, } \\
\text { alpha }\end{array}$ & 17 & 3 \\
\hline & $C D K 1$ & CDK1 & Cyclin-dependent kinase 1 & 10 & 7 \\
\hline & $C D K 5$ & CDK5 & Cyclin-dependent kinase 5 & 7 & 2 \\
\hline & $D A P 3$ & DAP3 & Death associated protein 3 & 1 & 1 \\
\hline & $G D A P 1$ & GDAP1 & Ganglioside-induced differentiation-associated protein 1 & 8 & 5 \\
\hline & GHITM & MICS1 & Growth hormone inducible transmembrane protein & 10 & 2 \\
\hline & MTFPl & MTFP1 & Mitochondrial fission process 1 & 22 & 2 \\
\hline & MUL1 & MAPL & Mitochondrial E3 ubiquitin protein ligase 1 & 1 & 0 \\
\hline & PPP3CA & Calcineurin & Protein phosphatase 3 , catalytic subunit, alpha isozyme & 4 & 47 \\
\hline & $P P P 3 C B$ & Calcineurin & Protein phosphatase 3 , catalytic subunit, beta isozyme & 10 & 1 \\
\hline & РPР3CC & Calcineurin & Protein phosphatase 3, catalytic subunit, gamma isozyme & 8 & 10 \\
\hline & $P R K A C A$ & PKA & Protein kinase, cAMP-dependent, catalytic, alpha & 19 & 0 \\
\hline & $P R K A C B$ & PKA & Protein kinase, cAMP-dependent, catalytic, beta & 1 & 10 \\
\hline & $P R K A C G$ & PKA & Protein kinase, cAMP-dependent, catalytic, gamma & 9 & 3 \\
\hline & $R A B 32$ & Rab32 & RAB32, member RAS oncogene family & 6 & 1 \\
\hline & SENP5 & SENP5 & SUMO1/sentrin specific protease 5 & 3 & 0 \\
\hline & SH3GLB1 & $\begin{array}{l}\text { Bif-1/ } \\
\text { Endophilin B1 }\end{array}$ & SH3-domain GRB2-like endophilin B1 & 1 & 2 \\
\hline & SMCR7 & MiD49 & Mitochondrial dynamics protein of $49 \mathrm{kDa}$ & 17 & 0 \\
\hline & $S M C R 7 L$ & MiD51 & Mitochondrial dynamics protein of $51 \mathrm{kDa}$ & 22 & 0 \\
\hline & SUMO1 & SUMO1 & SMT3 suppressor of mif two 3 homolog 1 & 2 & 0 \\
\hline
\end{tabular}


Table 1. Information about the SNPs in the mitochondrial dynamics regulating genes (Continued)

\begin{tabular}{|c|c|c|c|c|c|}
\hline Function & Gene & Gene product & Gene description & Chr. & $\begin{array}{l}\text { No. of } \\
\text { SNPs }\end{array}$ \\
\hline \multirow[t]{3}{*}{ Fission-regulation } & TIMM $8 A$ & DDP/Timm8a & $\begin{array}{l}\text { Translocase of inner mitochondrial membrane } 8 \\
\text { homolog A }\end{array}$ & $\mathrm{X}$ & 1 \\
\hline & TMEM11 & TMEM11/PMI & Transmembrane protein 11 & 17 & 1 \\
\hline & USP30 & USP30 & Ubiquitin specific peptidase 30 & 12 & 2 \\
\hline \multirow{5}{*}{$\begin{array}{l}\text { Fusion- and } \\
\text { fission-regulation }\end{array}$} & $B A X$ & Bax & BCL2-associated X protein & 19 & 1 \\
\hline & $B A K 1$ & Bak & BCL2-antagonist/killer 1 & 6 & 2 \\
\hline & MARCH5 & $\begin{array}{l}\text { MARCH-V/ } \\
\text { MITOL }\end{array}$ & Membrane-associated ring finger $(\mathrm{C} 3 \mathrm{HC} 4) 5$ & 10 & 4 \\
\hline & PINK1 & Pink1 & PTEN induced putative kinase 1 & 1 & 8 \\
\hline & PARK2 & Parkin & Parkinson disease 2, parkin & 6 & 227 \\
\hline
\end{tabular}

Abbreviation: Chr., chromosome. *The SNPs were selected from the KARE data based on their locations within the gene boundary $(5 \mathrm{~kb}$ upstream and downstream of the first and last exons, respectively) according to NCBI human genome build 36.

Table 2. Basic characteristics of the subjects in the KARE study cohort

\begin{tabular}{lcccc}
\hline \hline \multicolumn{1}{c}{ Characteristics } & Total & Males & Females & $P$ value \\
\hline Number of subjects & 7551 & 3747 & 3804 & \\
Age $(M$ years $\pm \mathrm{SD})$ & $51.44 \pm 8.78$ & $52.17 \pm 8.68$ & $51.59 \pm 8.89$ & 0.113 \\
Body mass index $(\mathrm{BMI})\left(M \mathrm{~kg} / \mathrm{m}^{2} \pm \mathrm{SD}\right)$ & $24.42 \pm 3.07$ & $24.14 \pm 2.90$ & $24.68 \pm 3.22$ & $<0.0001$ \\
Fasting blood glucose $(M \mathrm{mg} / \mathrm{dl} \pm \mathrm{SD})$ & $87.21 \pm 21.51$ & $89.89 \pm 23.29$ & $84.58 \pm 19.24$ & $<0.0001$ \\
\hline
\end{tabular}

Abbreviations: $\mathrm{M}$, mean value; SD, standard deviation. *Significant differences in characteristics between the males and females subjects were determined by the two-tailed Student's $t$-test.

Among the 47 genes, 36 genes had SNPs, which were 416 SNPs based on KARE genotype data (Table 1). Among the 36 genes, 10 genes had only one SNP each and the rest 26 genes had more than two SNPs. In particular, 227 SNPs were available in PARK2.

\section{Basic subject characteristics}

Basic characteristics of the 7,551 subjects in the KARE study cohort are shown in Table 1. The subjects provided the glucose level showed following characteristics: sex ratio was approximately equal, the mean age was $51.44 \pm 8.78$ years, mean BMI and fasting blood glucose were $24.42 \pm$ $3.07 \mathrm{~kg} / \mathrm{m}^{2}$ and $87.21 \pm 21.51 \mathrm{mg} / \mathrm{dL}$, respectively. Significant differences between the males and females in BMI and fasting blood glucose were observed as determined by the two-tailed Student's $t$-test. In females, higher value of BMI and lower value of fasting blood glucose were observed compared with those of males (Table 2).

\section{Association between the SNPs from PRKACB, PPP3CA, $P A R K 2$ and $G D A P 1$ genes and the fasting blood glucose} levels

We conducted statistical analyses toward the 416 SNPs of 36 genes to investigate quantitative association with fasting blood glucose levels of the KARE subjects. Through the analyses, 4 SNPs of PRKACB, 13 SNPs of PPP3CA, 6 SNPs of $P A R K 2$, and 3 SNPs of GDAP1 were found to have significant association with the fasting blood glucose level (Table 3). The SNPs of the rest 32 genes were not associated with the fasting blood glucose level. Minor allele frequency, genotype counts, genotype glucose, effect size and $P$ values of the associated SNPs were listed in Table 3. The three associated genes (PRKACB, PPP3CA, and GDAPl) have been known to have functions for fission-regulation in mito- 
Table 3. The significant SNPs in the mitochondrial dynamics regulating genes associated with fasting blood glucose levels in the KARE study cohort

\begin{tabular}{|c|c|c|c|c|c|c|c|c|c|c|c|c|}
\hline \multirow[b]{2}{*}{ Gene } & \multirow[b]{2}{*}{ SNP } & \multirow[b]{2}{*}{$\mathrm{A} 1$} & \multirow[b]{2}{*}{ MAF } & \multirow{2}{*}{$\begin{array}{l}\text { Function \& } \\
\text { RegulomeDB } \\
\text { results }\end{array}$} & \multicolumn{3}{|c|}{ Genotype counts } & \multicolumn{3}{|c|}{ Genotype glucose $(M \mathrm{mg} / \mathrm{dl} \pm \mathrm{SD})$} & \multicolumn{2}{|c|}{ Additive model } \\
\hline & & & & & $\mathrm{A} 1 / \mathrm{A} 1$ & $\mathrm{~A} 1 / \mathrm{A} 2$ & $\mathrm{~A} 2 / \mathrm{A} 2$ & $\mathrm{~A} 1 / \mathrm{A} 1$ & $\mathrm{~A} 1 / \mathrm{A} 2$ & $\mathrm{~A} 2 / \mathrm{A} 2$ & $\begin{array}{l}\text { Effect } \\
\text { size }\end{array}$ & $P$ value \\
\hline \multirow[t]{4}{*}{$P R K A C B$} & rs6701486 & $\mathrm{C}$ & 0.409 & Intron, 7 & 1213 & 3562 & 2593 & $\begin{array}{r}86.78 \\
+\quad 20.17\end{array}$ & $\begin{array}{r}86.64 \\
+\quad 20.78\end{array}$ & $\begin{array}{r}88.21 \\
\pm \quad 23.03\end{array}$ & $\begin{array}{r}-1.03 \\
\pm 0.36\end{array}$ & $3.8 \times 10^{-3}$ \\
\hline & rs2642186 & $\mathrm{C}$ & 0.488 & Intron, 6 & 1747 & 3678 & 1939 & $\begin{array}{r}87.04 \\
+20.49\end{array}$ & $\begin{array}{r}86.62 \\
+\quad 20.71\end{array}$ & $\begin{array}{r}88.48 \\
+23.76\end{array}$ & $\begin{array}{r}-0.81 \\
\pm 0.35\end{array}$ & 0.021 \\
\hline & rs 12723299 & A & 0.320 & Intron, 5 & 740 & 3207 & 3421 & $\begin{array}{r}86.58 \\
+20.95\end{array}$ & $\begin{array}{r}86.66 \\
\pm \quad 20.74\end{array}$ & $\begin{array}{r}87.87 \\
+22.31\end{array}$ & $\begin{array}{r}-0.99 \\
\pm 0.38\end{array}$ & $8.5 \times 10^{-3}$ \\
\hline & rs2134648 & $\mathrm{T}$ & 0.322 & Intron, 7 & 745 & 3224 & 3390 & $\begin{array}{r}86.55 \\
+20.89 \\
\end{array}$ & $\begin{array}{r}86.65 \\
\pm \quad 20.74 \\
\end{array}$ & $\begin{array}{r}87.91 \\
\pm 22.36 \\
\end{array}$ & $\begin{array}{r}-1.04 \\
\pm 0.38\end{array}$ & $5.9 \times 10^{-3}$ \\
\hline \multirow[t]{13}{*}{ PPP3CA } & rs3804359 & G & 0.045 & Intron, 7 & 27 & 606 & 6731 & $\begin{array}{r}103.10 \\
\pm \quad 54.28\end{array}$ & $\begin{array}{r}88.02 \\
+\quad 26.94\end{array}$ & $\begin{array}{r}87.07 \\
+20.70\end{array}$ & $\begin{array}{r}2.11 \\
\pm \quad 0.83\end{array}$ & 0.011 \\
\hline & rs2850359 & $\mathrm{T}$ & 0.340 & Intron, 5 & 878 & 3285 & 3205 & $\begin{array}{r}86.65 \\
\pm \quad 18.18\end{array}$ & $\begin{array}{r}86.74 \\
\pm \quad 19.47\end{array}$ & $\begin{array}{r}87.86 \\
\pm \quad 24.17\end{array}$ & $\begin{array}{r}-0.75 \\
\pm 0.37\end{array}$ & 0.041 \\
\hline & rs3804406 & A & 0.335 & Intron, 7 & 857 & 3253 & 3256 & $\begin{array}{r}86.71 \\
\pm \quad 18.24\end{array}$ & $\begin{array}{r}86.72 \\
\pm \quad 19.55\end{array}$ & $\begin{array}{r}87.85 \\
\pm \quad 24.02\end{array}$ & $\begin{array}{r}-0.74 \\
\pm 0.37\end{array}$ & 0.044 \\
\hline & rs3804408 & $\mathrm{C}$ & 0.340 & Intron, 6 & 877 & 3286 & 3203 & $\begin{array}{r}86.65 \\
\pm \quad 18.19\end{array}$ & $\begin{array}{r}86.73 \\
\pm \quad 19.47\end{array}$ & $\begin{array}{r}87.86 \\
\pm 24.17\end{array}$ & $\begin{array}{r}-0.75 \\
\pm 0.37\end{array}$ & 0.039 \\
\hline & rs2583405 & A & 0.355 & Intron, 6 & 955 & 3344 & 3065 & $\begin{array}{r}86.87 \\
+\quad 18.45\end{array}$ & $\begin{array}{r}86.66 \\
\pm \quad 19.47\end{array}$ & $\begin{array}{r}87.93 \\
\pm \quad 24.33\end{array}$ & $\begin{array}{r}-0.72 \\
\pm 0.36\end{array}$ & 0.047 \\
\hline & rs 2850963 & $\mathrm{C}$ & 0.458 & Intron, 1f & 1566 & 3624 & 2175 & $\begin{array}{r}87.19 \\
\pm \quad 19.52\end{array}$ & $\begin{array}{r}86.46 \\
\pm \quad 19.04\end{array}$ & $\begin{array}{r}88.49 \\
\pm \quad 26.21\end{array}$ & $\begin{array}{r}-0.70 \\
\pm 0.35\end{array}$ & 0.045 \\
\hline & rs2850336 & A & 0.341 & Intron, 6 & 880 & 3295 & 3191 & $\begin{array}{r}86.62 \\
\pm \quad 18.17\end{array}$ & $\begin{array}{r}86.73 \\
\pm \quad 19.45\end{array}$ & $\begin{array}{r}87.89 \\
+\quad 24.21\end{array}$ & $\begin{array}{r}-0.78 \\
\pm 0.37\end{array}$ & 0.034 \\
\hline & rs1441433 & $\mathrm{T}$ & 0.460 & Intron, 1f & 1599 & 3585 & 2164 & $\begin{array}{r}87.02 \\
\pm \quad 19.23\end{array}$ & $\begin{array}{r}86.53 \\
\pm \quad 19.25\end{array}$ & $\begin{array}{r}88.43 \\
\pm \quad 25.93\end{array}$ & $\begin{array}{r}-0.76 \\
\pm 0.35\end{array}$ & 0.029 \\
\hline & rs1530259 & $\mathrm{C}$ & 0.463 & Intron, 6 & 1611 & 3613 & 2123 & $\begin{array}{r}86.77 \\
+\quad 19.04\end{array}$ & $\begin{array}{r}86.86 \\
+20.17\end{array}$ & $\begin{array}{r}88.19 \\
+25.23\end{array}$ & $\begin{array}{r}-0.74 \\
\pm 0.35\end{array}$ & 0.033 \\
\hline & rs 2850370 & G & 0.461 & Intron, 6 & 1606 & 3609 & 2151 & $\begin{array}{r}86.73 \\
+\quad 18.99\end{array}$ & $\begin{array}{r}86.86 \\
\pm \quad 20.12\end{array}$ & $\begin{array}{r}88.19 \\
+25.20\end{array}$ & $\begin{array}{r}-0.77 \\
\pm 0.35\end{array}$ & 0.027 \\
\hline & rs2583399 & A & 0.461 & Intron, 1f & 1609 & 3606 & 2152 & $\begin{array}{r}86.71 \\
\pm \quad 18.99\end{array}$ & $\begin{array}{r}86.85 \\
+\quad 20.13\end{array}$ & $\begin{array}{r}88.20 \\
\pm \quad 25.20\end{array}$ & $\begin{array}{r}-0.78 \\
\pm 0.35\end{array}$ & 0.024 \\
\hline & rs2850371 & $\mathrm{C}$ & 0.464 & Intron, 6 & 1623 & 3614 & 2128 & $\begin{array}{r}86.75 \\
\pm \quad 18.98\end{array}$ & $\begin{array}{r}86.84 \\
\pm \quad 20.14\end{array}$ & $\begin{array}{r}88.22 \\
\pm \quad 25.24\end{array}$ & $\begin{array}{r}-0.77 \\
\pm 0.35\end{array}$ & 0.026 \\
\hline & rs2583394 & G & 0.464 & Intron, 1f & 1631 & 3607 & 2129 & $\begin{array}{r}86.74 \\
+\quad 18.95\end{array}$ & $\begin{array}{r}86.83 \\
\pm \quad 20.15\end{array}$ & $\begin{array}{r}88.23 \\
+\quad 25.24\end{array}$ & $\begin{array}{r}-0.79 \\
\pm 0.35\end{array}$ & 0.023 \\
\hline \multirow[t]{6}{*}{ PARK2 } & rs2209247 & $\mathrm{T}$ & 0.044 & Intron, 5 & 13 & 620 & 6735 & $\begin{array}{r}87.00 \\
\pm \quad 15.53\end{array}$ & $\begin{array}{r}89.02 \\
+\quad 26.50\end{array}$ & $\begin{array}{r}87.05 \\
\pm 21.00\end{array}$ & $\begin{array}{r}1.71 \\
\pm 0.85\end{array}$ & 0.046 \\
\hline & rs 10455889 & G & 0.466 & Intron, 7 & 1622 & 3627 & 2118 & $\begin{array}{r}86.22 \\
\pm \quad 18.68\end{array}$ & $\begin{array}{r}86.91 \\
\pm \quad 20.32\end{array}$ & $\begin{array}{r}88.50 \\
\pm \quad 25.16\end{array}$ & $\begin{array}{r}-1.14 \\
\pm 0.35\end{array}$ & $1.1 \times 10^{-3}$ \\
\hline & rs9365294 & G & 0.467 & Intron, 7 & 1625 & 3630 & 2113 & $\begin{array}{r}86.12 \\
+18.43\end{array}$ & $\begin{array}{r}86.97 \\
\pm \quad 20.45\end{array}$ & $\begin{array}{r}88.47 \\
+\quad 25.14\end{array}$ & $\begin{array}{r}-1.15 \\
\pm 0.35\end{array}$ & $9.1 \times 10^{-4}$ \\
\hline & rs9356011 & $\mathrm{T}$ & 0.205 & Intron, 7 & 333 & 2349 & 4686 & $\begin{array}{r}88.05 \\
\pm \quad 29.70\end{array}$ & $\begin{array}{r}87.89 \\
+21.92\end{array}$ & $\begin{array}{r}86.81 \\
\pm \quad 20.59\end{array}$ & $\begin{array}{r}0.88 \\
\pm 0.43\end{array}$ & 0.041 \\
\hline & rs9364660 & G & 0.489 & Intron, 4 & 1770 & 3662 & 1935 & $\begin{array}{r}86.65 \\
+19.48\end{array}$ & $\begin{array}{r}86.84 \\
+\quad 19.88\end{array}$ & $\begin{array}{r}88.43 \\
+\quad 25.79\end{array}$ & $\begin{array}{r}-0.84 \\
\pm 0.35\end{array}$ & 0.015 \\
\hline & rs 7758475 & $\mathrm{C}$ & 0.459 & Intron, 5 & 1551 & 3654 & 2159 & $\begin{array}{r}86.79 \\
\pm \quad 19.86\end{array}$ & $\begin{array}{r}86.80 \\
+\quad 19.69\end{array}$ & $\begin{array}{r}88.21 \\
\pm \quad 25.26\end{array}$ & $\begin{array}{r}-0.70 \\
\pm 0.35\end{array}$ & 0.047 \\
\hline
\end{tabular}


Table 3. The significant SNPs in the mitochondrial dynamics regulating genes associated with fasting blood glucose levels in the KARE study cohort (Continued)

\begin{tabular}{|c|c|c|c|c|c|c|c|c|c|c|c|c|}
\hline \multirow[b]{2}{*}{ Gene } & \multirow[b]{2}{*}{ SNP } & \multirow[b]{2}{*}{$\mathrm{A} 1$} & \multirow[b]{2}{*}{ MAF } & \multirow{2}{*}{$\begin{array}{l}\text { Function \& } \\
\text { RegulomeDB } \\
\text { results }\end{array}$} & \multicolumn{3}{|c|}{ Genotype counts } & \multicolumn{3}{|c|}{ Genotype glucose $(M \mathrm{mg} / \mathrm{dl} \pm \mathrm{SD})$} & \multicolumn{2}{|c|}{ Additive model } \\
\hline & & & & & $\mathrm{A} 1 / \mathrm{A} 1$ & $\mathrm{~A} 1 / \mathrm{A} 2$ & $\mathrm{~A} 2 / \mathrm{A} 2$ & $\mathrm{~A} 1 / \mathrm{A} 1$ & $\mathrm{~A} 1 / \mathrm{A} 2$ & $\mathrm{~A} 2 / \mathrm{A} 2$ & $\begin{array}{c}\text { Effect } \\
\text { size }\end{array}$ & $P$ value \\
\hline \multirow[t]{3}{*}{$G D A P 1$} & rs6993852 & G & 0.280 & Downstream, 6 & 615 & 2927 & 3824 & $\begin{array}{r}87.40 \\
+\quad 20.69\end{array}$ & $\begin{array}{r}88.17 \\
+\quad 23.95\end{array}$ & $\begin{array}{r}86.46 \\
\pm \quad 19.55\end{array}$ & $\begin{array}{r}0.90 \\
\pm 0.38\end{array}$ & 0.019 \\
\hline & rs4738451 & A & 0.279 & Downstream, 7 & 621 & 2880 & 3823 & $\begin{array}{r}87.38 \\
\pm \quad 20.61\end{array}$ & $\begin{array}{r}88.10 \\
\pm \quad 23.92\end{array}$ & $\begin{array}{r}86.45 \\
+19.56\end{array}$ & $\begin{array}{r}0.88 \\
\pm 0.38\end{array}$ & 0.022 \\
\hline & rs4738452 & $\mathrm{C}$ & 0.281 & Downstream, 7 & 619 & 2927 & 3816 & $\begin{array}{r}87.34 \\
+\quad 20.65\end{array}$ & $\begin{array}{r}88.17 \\
+\quad 23.95\end{array}$ & $\begin{array}{r}86.47 \\
+\quad 19.57\end{array}$ & $\begin{array}{r}0.88 \\
+0.38\end{array}$ & 0.021 \\
\hline
\end{tabular}

Age, gneder, body mass index (BMI) and cohorts were included as covariates in additive genetic model. Abbreviatins: A1, minor allele; A2, major allele; $\mathrm{M}$ mean value; MAF, minor allele frequency; SD, standard deviation. RegulomeDB score were presented the changes by SNP such as 1f: eQTL+TF binding DNase peak, 4: TF binding+DNase peak, 5: TF binding or DNase peak, and 6: other and 7: none.

chondrial dynamics, and PARK2 gene has been known to have function for fusion- and fission-regulation (Table 1). The ratio of significantly associated SNPs out of total analyzed SNPs were $40 \%$ in $P R K A C B$ (4 among the 10 SNPs), $27.7 \%$ in PPP3CA (13 among the 47 SNPs), 2.64\% in PARK2 (6 among the 227 SNPs), and $60 \%$ in GDAPl (3 among the 5 SNPs). The minor allele of 4 SNPs in PRKACB were uniformly associated with lower glucose levels, and the minor allele of 3 SNPS in GDAPl were uniformly associated with higher glucose levels (Table 3 ).

\section{DISCUSSION}

In this study, we used 416 SNPs of 36 genes that are related to mitochondrial dynamics or shaping and found 26 SNPs of 4 genes had significant association with fasting blood glucose levels. The 4 genes are $P R K A C B, P P P 3 C A$, $P A R K 2$ and GDAP1. The PRKACB (protein kinase, cAMPdependent, catalytic, beta) gene located on chromosome 1p36.1 encodes a catalytic subunit of cAMP (cyclic AMP)dependent protein kinase, which mediates signaling through cAMP. And the cAMP signaling is important to a number of processes, including cell proliferation and differentiation (Gamm et al., 1996). The PRKACB are involved in the regulation of lipid and glucose metabolism as well as regulation of fission in the mitochondrial dynamics (Otera et al., 2013). But, there is no published article yet that showed association of SNPs of PRKACB with glucose or type 2 diabetes.
The PPP3CA (protein phosphatase 3 catalytic subunit alpha) gene located on chromosome 4q24 encodes calcineurin isozyme. Calcineurin is a calcium and calmodulin dependent serine/threonine protein phosphatase, and also involved in fission regulation of mitochondrial shaping (Otera et al., 2013). There is one report showed association of the genetic variation of $P P P 3 C A$ with type 2 diabetes (Diabetes Genetics Initiative of Broad Institute of Harvard et al., 2007).

The PARK2 (parkin RBR E3 ubiquitin protein ligase) gene located on chromosome 6q25.2-q27 encodes a component of a multiprotein E3 ubiquitin ligase complex that mediates the targeting of substrate proteins for proteasomal degradation. Mutations in this gene are known to cause Parkinson disease and autosomal recessive juvenile Parkinson disease related to mitochondrial dysfunction (Hang et al., 2015). Moreover, there are many reports that $P A R K 2$ gene associated with various metabolic traits including insulin-related traits (Ober et al., 2009; Pollin et al., 2008; Hiura et al., 2010; Paternoster et al., 2011; Jin et al., 2014).

The GDAP1 (ganglioside induced differentiation associated protein 1) gene located on chromosome 8q21.11 encodes a member of the ganglioside-induced differentiation-associated protein family, which may play a role in a signal transduction pathway during neuronal development. Mutations in this gene have been known to associate with various forms of Charcot-Marie-Tooth Disease and neuropathy related to mitochondrial fission (Kabzinska et al., 2014; Huber et al., 2013). There is limited article that showed association of GDAP1 gene with the obesity-related traits (Fox et al., 2007). 
It has been known that mitochondrial dynamics genes are involved in glucose metabolism. In this study, association of the genetic variants of the mitochondrial dynamics genes with fasting blood glucose level was newly analyzed. We were able to find that 4 genes ( $P R K A C B, P P P 3 C A, P A R K 2$, and $G D A P 1)$ among the mitochondrial dynamics genes might have more relationship with fasting blood glucose level the other genes. Interestingly, 3 genes ( $P R K A C B, P P P 3 C A$, and $G D A P 1)$ are involved in fission-regulation which suggests that genes for regulating mitochondrial fission may have more roles for regulating blood glucose.

There are some limitations to this current study. Since $\mathrm{KARE}$ is a pre-genotyping data for genome-wide association study, the 36 genes had available SNPs from the candidate 46 genes for mitochondrial dynamics. Therefore, it may be difficult to reach a definitive conclusion for all of the mitochondrial dynamics genes at this time. We hope to examine the association of the 10 unanalyzed genes when the new genotyping data becomes available. Although limited number of the genes was involved, this study presents a systemic approach concerning mitochondrial dynamics with blood glucose level.

\section{Acknowledgements}

This research was supported by the Academic Research fund of Hoseo University in 2015 (2015-0092).

\section{Conflict of interest}

The authors declare that they have no competing interests.

\section{REFERENCES}

Benard G, Karbowski M. Mitochondrial fusion and division: Regulation and role in cell viability. Semin Cell Dev Biol. 2009. 20: $365-374$

Cerveny KL, Tamura Y, Zhang Z, Jensen RE, Sesaki H. Regulation of mitochondrial fusion and division. Trends Cell Biol. 2007. 17: 563-569.

Chan DC. Mitochondrial fusion and fission in mammals. Annu Rev Cell Dev Biol. 2006. 22: 79-99.

Cho YS, Go MJ, Kim YJ, Heo JY, Oh JH, Ban HJ, Yoon D, Lee MH, Kim DJ, Park M, Cha SH, Kim JW, Han BG, Min H, Ahn Y, Park MS, Han HR, Jang HY, Cho EY, Lee JE, Cho
NH, Shin C, Park T, Park JW, Lee JK, Cardon L, Clarke G, McCarthy MI, Lee JY, Lee JK, Oh B, Kim HL. A large-scale genome-wide association study of Asian populations uncovers genetic factors influencing eight quantitative traits. Nat Genet. 2009. 41: 527-534.

Diabetes Genetics Initiative of Broad Institute of Harvard and MIT, Lund University, Novartis Institutes of BioMedical Research. Genome-wide association analysis identifies loci for type 2 diabetes and triglyceride levels. Science. 2007. 316: 1331 $-1336$.

Fox CS, Heard-Costa N, Cupples LA, Dupuis J, Vasan RS, Atwood LD. Genome-wide association to body mass index and waist circumference: The Framingham heart study $100 \mathrm{~K}$ project. BMC Med Genet. 2007. 8 Suppl 1: S18.

Gamm DM, Baude EJ, Uhler MD. The major catalytic subunit isoforms of cAMP-dependent protein kinase have distinct biochemical properties in vitro and in vivo. J Biol Chem. 1996. 271: 15736-15742.

Hang L, Thundyil J, Lim KL. Mitochondrial dysfunction and parkinson disease: A parkin-AMPK alliance in neuroprotection. Ann N Y Acad Sci. 2015. 1350: 37-47.

Hiura Y, Tabara Y, Kokubo Y, Okamura T, Miki T, Tomoike H, Iwai N. A genome-wide association study of hypertensionrelated phenotypes in a Japanese population. Circ J. 2010. 74: 2353-2359.

Hoppins S, Lackner L, Nunnari J. The machines that divide and fuse mitochondria. Annu Rev Biochem. 2007. 76: 751-780.

Huber N, Guimaraes S, Schrader M, Suter U, Niemann A. CharcotMarie-tooth disease-associated mutants of GDAP1 dissociate its roles in peroxisomal and mitochondrial fission. EMBO Rep. 2013. 14: 545-552

In SK, Hyun TK, Kim E-J, Lee EJ. A comparative study of the concentration of salivary and blood glucose in normal and diabetic subjects. Journal of Experimental \& Biomedical Sciences. 2013. 19: 105-111.

Jin HS, Kim J, Lee SJ, Kim K, Go MJ, Lee JY, Lee HJ, Song J, Jeon BT, Roh GS, Kim SJ, Kim BY, Hong KW, Yoo YH, Oh B, Kang Y, Jeong SY. The PARK2 gene is involved in the maintenance of pancreatic beta-cell functions related to insulin production and secretion. Mol Cell Endocrinol. 2014. 382: 178-189.

Jin HJ, Kim J, Kyung TK, Ho JS. Application of an efficient method for isolation of mitochondria from biological samples. Biomedical Science Letters. 2014. 20: 180-184.

Kabzinska D, Kotruchow K, Cegielska J, Hausmanowa-Petrusewicz 
I, Kochanski A. A severe recessive and a mild dominant form of Charcot-Marie-tooth disease associated with a newly identified Glu222Lys GDAP1 gene mutation. Acta Biochim Pol. 2014. 61: 739-744.

Mason CC, Hanson RL, Knowler WC. Progression to type 2 diabetes characterized by moderate then rapid glucose increases. Diabetes. 2007. 56: 2054-2061.

McBride HM, Neuspiel M, Wasiak S. Mitochondria: More than just a powerhouse. Curr Biol. 2006. 16: R551-560.

Ober C, Nord AS, Thompson EE, Pan L, Tan Z, Cusanovich D, Sun Y, Nicolae R, Edelstein C, Schneider DH, Billstrand C, Pfaffinger D, Phillips N, Anderson RL, Philips B, Rajagopalan R, Hatsukami TS, Rieder MJ, Heagerty PJ, Nickerson DA, Abney M, Marcovina S, Jarvik GP, Scanu AM, Nicolae DL. Genome-wide association study of plasma lipoprotein (a) levels identifies multiple genes on chromosome 6q. J Lipid Res. 2009. 50: 798-806.

Otera H, Ishihara N, Mihara K. New insights into the function and regulation of mitochondrial fission. Biochim Biophys Acta. 2013. 1833: 1256-1268.

Paternoster L, Evans DM, Nohr EA, Holst C, Gaborieau V, Brennan P, Gjesing AP, Grarup N, Witte DR, Jorgensen T, Linneberg A, Lauritzen T, Sandbaek A, Hansen T, Pedersen O, Elliott KS, Kemp JP, St Pourcain B, McMahon G, Zelenika D, Hager J, Lathrop M, Timpson NJ, Smith GD, Sorensen TI. Genomewide population-based association study of extremely overweight young adults--the GOYA study. PLoS One. 2011. 6: e24303.

Pollin TI, Damcott CM, Shen H, Ott SH, Shelton J, Horenstein RB, Post W, McLenithan JC, Bielak LF, Peyser PA, Mitchell BD, Miller M, O'Connell JR, Shuldiner AR. A null mutation in human APOC3 confers a favorable plasma lipid profile and apparent cardioprotection. Science. 2008. 322: 1702-1705.
Prokopenko I, Langenberg C, Florez JC, Saxena R, Soranzo N, Thorleifsson G, Loos RJ, Manning AK, Jackson AU, Aulchenko Y, Potter SC, Erdos MR, Sanna S, Hottenga JJ, Wheeler E, Kaakinen M, Lyssenko V, Chen WM, Ahmadi K, Beckmann JS, Bergman RN, Bochud M, Bonnycastle LL, Buchanan TA, Cao A, Cervino A, Coin L, Collins FS, Crisponi L, de Geus EJ, Dehghan A, Deloukas P, Doney AS, Elliott P, Freimer N, Gateva V, Herder C, Hofman A, Hughes TE, Hunt $\mathrm{S}$, Illig $\mathrm{T}$, Inouye $\mathrm{M}$, Isomaa $\mathrm{B}$, Johnson $\mathrm{T}$, Kong A, Krestyaninova M, Kuusisto J, Laakso M, Lim N, Lindblad U, Lindgren CM, McCann OT, Mohlke KL, Morris AD, Naitza S, Orru M, Palmer CN, Pouta A, Randall J, Rathmann W, Saramies J, Scheet P, Scott LJ, Scuteri A, Sharp S, Sijbrands E, Smit JH, Song K, Steinthorsdottir V, Stringham HM, Tuomi T, Tuomilehto J, Uitterlinden AG, Voight BF, Waterworth D, Wichmann HE, Willemsen G, Witteman JC, Yuan X, Zhao JH, Zeggini E, Schlessinger D, Sandhu M, Boomsma DI, Uda M, Spector TD, Penninx BW, Altshuler D, Vollenweider P, Jarvelin MR, Lakatta E, Waeber G, Fox CS, Peltonen L, Groop LC, Mooser V, Cupples LA, Thorsteinsdottir U, Boehnke M, Barroso I, Van Duijn C, Dupuis J, Watanabe RM, Stefansson K, McCarthy MI, Wareham NJ, Meigs JB, Abecasis GR. Variants in MTNR1B influence fasting glucose levels. Nat Genet. 2009. 41: 77-81.

Rabbee N, Speed TP. A genotype calling algorithm for affymetrix SNP arrays. Bioinformatics. 2006. 22: 7-12.

Watanabe RM, Valle T, Hauser ER, Ghosh S, Eriksson J, Kohtamaki K, Ehnholm C, Tuomilehto J, Collins FS, Bergman RN, Boehnke M. Familiality of quantitative metabolic traits in finnish families with non-insulin-dependent diabetes mellitus. Finland-United States investigation of NIDDM genetics (FUSION) study investigators. Hum Hered. 1999. 49: 159168. 